Rianne J.M. Lammers, Wim P.J. Witjes, and J. Alfred Witjes, Radboud University Nijmegen Medical Centre, Nijmegen; Wim P.J. Witjes, Maria H.D. Janzing-Pastors, and Christien T.M Caris, CuraTrial Site Management Organisation \& Research, Arnhem, the Netherlands.

Submitted September 1, 2011; accepted March 2, 2012; published online ahead of print at www.jco.org on May 14, 2012

Supported by a grant from biosyn Arzneimittel $\mathrm{GmbH}$ to CuraTrial Site Management Organisation \& Research.

All authors had access to all the data in the study and take responsibility for the integrity of the data and the accuracy of the data analysis. This trial was not officially registered, because registration was not required when this trial started (2002).

Authors' disclosures of potential conflicts of interest and author contributions are found at the end of this article.

Corresponding author: J. Alfred Witjes, $\mathrm{MD}, \mathrm{PhD}$, Radboud University Nijmegen Medical Centre, Department of Urology, Geert Grooteplein South 10 (659), PO Box 9101, 6500 HB Nijmegen, the Netherlands; e-mail: f.witjes@uro.umcn.nl.

C 2012 by American Society of Clinical Oncology

0732-183X/12/3018-2273/\$20.00 DOI: $10.1200 / J C 0.2011 .39 .2936$

\title{
Intracutaneous and Intravesical Immunotherapy With Keyhole Limpet Hemocyanin Compared With Intravesical Mitomycin in Patients With Non-Muscle-Invasive Bladder Cancer: Results From a Prospective Randomized Phase III Trial
}

Rianne J.M. Lammers, Wim P.J. Witjes, Maria H.D. Janzing-Pastors, Christien T.M. Caris, and J. Alfred Witjes

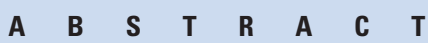

\section{Purpose}

Despite current treatment after transurethral resection of a bladder tumor, recurrences and progression remain a problem. Keyhole limpet hemocyanin $(K L H)$ was beneficial in earlier studies. In this study, safety and efficacy of KLH were compared with that of mitomycin (MM).

\section{Patients and Methods}

Patients with intermediate- and high-risk non-muscle-invasive bladder cancer (NMIBC) without carcinoma in situ were enrolled in a randomized phase III trial. In all, 283 patients were randomly assigned for 16 adjuvant intravesical instillations with $\mathrm{KLH}$ after preimmunization, and 270 patients were randomly assigned for 11 adjuvant intravesical instillations with MM. Primary outcome measurement was recurrence-free survival (RFS). Secondary outcome measurements were progression-free survival, adverse events (AEs), and the effect of delayed-type hypersensitivity (DTH) response on clinical outcome.

\section{Results}

There were significantly more pT1 tumors in the MM group $(P=.01)$. In a log-rank test, univariate and multivariate Cox regression analysis, $\mathrm{KLH}$ was less effective than MM regarding RFS (all $P<$ .001). Progression was uncommon $(n=20)$. In univariate Cox regression analyses, $\mathrm{KLH}$ tended to prevent progression more effectively than $\mathrm{MM}$, but in multivariate Cox regression analyses, this could not be shown. AEs were common but mild. Fever, flu-like symptoms, and fatigue occurred significantly more after KLH treatment. Allergic reactions and other skin disorders occurred significantly more after MM treatment. Significantly more DTH-positive patients developed a recurrence than DTH-negative patients.

\section{Conclusion}

$\mathrm{KLH}$ had a different safety profile and was inferior to MM in preventing NMIBC recurrences. KLH tended to be more effective than $\mathrm{MM}$ in preventing progression. More research is needed to clarify the immunologic effects of $\mathrm{KLH}$ and the effects of $\mathrm{KLH}$ on progression.

\section{J Clin Oncol 30:2273-2279. (C) 2012 by American Society of Clinical Oncology}

\section{INTRODUCTION}

Worldwide, about 386,300 new cases and 150,200 deaths from bladder urothelial cell carcinoma occurred in 2008. ${ }^{1}$ Approximately $70 \%$ is nonmuscle-invasive bladder cancer (NMIBC). ${ }^{2}$ The risk of recurrence and progression after treatment varies. Low-grade Ta NMIBC has a high chance of recurrence and a low chance of progression, whereas high-grade tumors have a high potential for malignancy. Treatment consists of transurethral resection of the bladder tumor (TURBT), followed by intravesical treatment. The choice of adjuvant treatment with either chemotherapy (eg, mitomycin [MM]) or immunotherapy (eg, Bacillus Calmette-Guérin [BCG]) depends on the risk group to which the patient belongs. ${ }^{3-6}$ Unfortunately, recurrences and adverse events (AEs) are common. ${ }^{7}$

Keyhole limpet hemocyanin (KLH), a glycoprotein from the gastropod mollusk Megathura crenulata is an immunocyanin that contributes to the uptake, transport, and release of oxygen during respiration. It also activates both the humoral and cellular immune systems, but the precise mechanism of action of KLH remains unknown. ${ }^{8}$ One commercially available intravesical immunocyanin is Immucothel (biosyn, Fellbach, Germany). 
Olsson et $\mathrm{al}^{9}$ were the first to report on $\mathrm{KLH}$ in 1974, with promising results on tumor recurrence. Results of other small phase II studies suggested a potential benefit of KLH over known treatments and a favorable safety profile. ${ }^{10-12}$ Our goal was to compare the efficacy and safety of KLH with that of MM in a prospective, randomized phase III trial.

\section{PATIENTS AND METHODS}

Eighteen Dutch institutions participated in this multicenter, prospective, randomized phase III trial. The study was conducted in compliance with Good Clinical Practice, the Declaration of Helsinki (version 1996), and with permission of all local ethical committees.

\section{Patient Selection}

Patients with a histologically proven $\mathrm{pTa} / 1$ grade 2 to 3 tumor, or with multiple or recurrent pTa grade 1 tumors, in whom complete TURBT was possible, were included after obtaining informed consent. Patients who received intravesical therapy within 6 months before entry or more than one instillation of MM within 2 years before inclusion were excluded. Patients with primary solitary pTa grade 1 tumors, carcinoma in situ (CIS), or tumors $\geq$ pT2 were also excluded. Other exclusion criteria were concurrent malignancy (except basal cell or squamous cell carcinoma of the skin), history of another malignancy with a disease-free interval $\leq 5$ years, expected poor compliance, WHO performance status more than 2 , uncontrollable urinary tract infection, any previous systemic cancer therapy or radiotherapy within 5 years, localization of tumor in prostatic urethra or upper urinary tract, pregnancy or lactation for women of reproductive age who refused to take adequate contraceptive measures, congenital or acquired immune deficiency syndromes, known hypersensitivity to $\mathrm{MM}$, and previous ( $\leq 1$ month) or current treatment with an investigational drug.

\section{Treatment Schedule}

After TURBT was complete and informed consent was signed, patients were randomly assigned for treatment with either KLH (Immucothel) or MM. The restricted blockwise procedure was used for random assignment.

In the KLH arm, patients started with preimmunization: intracutaneous (IC) injection of $1 \mathrm{mg} \mathrm{KLH}$ at intervals of 2 to 7 days until a delayed-type hypersensitivity (DTH) response was obtained. A real DTH response was defined as a palpable infiltrate in the skin. In case no DTH response developed, up to four IC injections were given. Intravesical treatment was started within 2 weeks after the TURBT, independent of the preimmunization result. If there was no DTH response after four injections, this was not a reason for discontinuing the treatment. Patients received a total of 16 intravesical instillations (20 mg KLH in $20 \mathrm{~mL}$ solvent) given once per week for 6 weeks and then once per month for 10 months (months 3, 4, 5, 6, 7, 8, 9, 10, 11 and 12).

In the MM arm, patients received a total of 11 instillations ( $40 \mathrm{mg} \mathrm{MM}$ in $50 \mathrm{mg}$ saline) given once per week for 4 weeks (weeks 1, 2, 3, and 4) and once per month for 5 months (months 2, 3, 4, 5, and 6) and then at 9 and 12 months.

\section{Follow-Up and Evaluation of Therapy}

Patients received urinary cytology and cystoscopy every 3 months for the first year, every 4 months during the second and third years, and every 6 months thereafter. All visible lesions were completely resected and confirmed by histologic examination. If a recurrence without progression was observed during the treatment period, the therapy was continued after complete TURBT. Patients went off-study in case of withdrawal of informed consent, second recurrence during treatment, first recurrence after completion of therapy, 5-year disease-free survival, occurrence of CIS, tumor of upper tract or prostatic urethra, or distant metastasis. Patients in whom a lesion was coagulated, not biopsied, or not treated at all continued in follow-up and were censored at the time of last follow-up.

\section{Objectives and Statistical Analysis}

The primary end point was the duration of recurrence-free survival (RFS). Secondary objectives were recurrence rate, number of recurrent tu- mors, duration of progression-free survival (PFS), AEs, and the effect of DTH response on clinical outcome. RFS was defined as the time interval between TURBT and time to first recurrence. Recurrence was defined as positive cystoscopy and histologically confirmed positive biopsies. Recurrence rate was defined as the number of cystoscopies at which recurrences were observed divided by the duration of follow-up in months multiplied by 100 . PFS was defined as the time interval between TURBT and time to progression. Progression was defined as recurrence with histologic pathology stage $\geq$ pT2, distant metastases, or death related to bladder cancer.

$\mathrm{KLH}$ is registered for prevention of bladder cancer after TURBT and after failure of established therapies. Therefore, a subgroup analysis was done in which RFS after KLH treatment was compared with MM treatment in patients with recurrence disease and a history of intravesical treatments.

Power analysis showed that 251 evaluable patients were needed in each treatment group to reject the hypothesis of noninferiority, at error rates of $\alpha / 2=.025$ and $\beta=.20$. With an expected dropout rate of $10 \%$, a total of 552 patients were needed in this study. The study was designed as a noninferiority study regarding RFS. Inferiority was defined as a ratio of median RFS ( $\mathrm{t}_{\mathrm{KLH}} /$ $\mathrm{t}_{\mathrm{MM}}$ ) more than 0.75 (eg, 1.31/1.75 years), assuming proportional hazards.

The Kaplan-Meier method and one-sided log-rank test were used to compare for differences in RFS and PFS between the two treatments. Crude hazard ratios (HRs) with univariate Cox regression were used to analyze the influence of patient and tumor characteristics separately on RFS and PFS. Adjusted HRs with multivariate Cox regression were used to correct for confounding factors. Patient and tumor characteristics were compared by using a two-sided $\chi^{2}$ test. Number of patients with a recurrence was compared by using a one-sided Fisher's exact test. Number of recurrent tumors was compared by using the one-sided Mann-Whitney $U$ test, and toxicity was compared by using the two-sided $\chi^{2}$ test. Effect of DTH response on outcome was analyzed with two-sided $\chi^{2}$ tests and log-rank tests. All two-sided analyses were performed at a $5 \%$ level of significance; all one-sided analyses were performed at a $2.5 \%$ level of significance. Statistical analyses were done with SPSS version 16.0 software (SPSS, Chicago, IL).

\section{RESULTS}

Between July 2003 and November 2007, 553 patients were randomly assigned: 283 patients to treatment with KLH and 270 to treatment with MM. For safety analysis, 537 patients were available: 273 patients in the KLH group and 264 patients in the MM group. A total of 272 patients received at least one KLH instillation, and 263 patients received at least one MM instillation. One patient was randomly assigned for KLH but received MM and was included in the MM group. One patient did not receive KLH treatment but experienced an AE before instillation, so he was included in the KLH group (Fig 1).

For the efficacy analyses, 267 patients in the KLH arm and 256 patients in the MM arm were included. In the KLH arm, 16 patients were excluded: 10 did not receive any instillation, one patient was treated with $\mathrm{MM}$, and five patients were ineligible (concurrent malignancy, $\mathrm{n}=3$; incomplete TURBT, $\mathrm{n}=2$ ). In the MM arm, 14 patients were excluded: seven did not receive any instillation, and seven were ineligible (concurrent malignancy, $\mathrm{n}=6$; tumor in prostatic urethra, $\mathrm{n}=1$; Fig 1).

\section{Patient and Tumor Characteristics}

Patient and tumor characteristics were comparable in both efficacy groups (Table 1), with the exception of tumor stage. Patients in the MM group had significantly more pT1 tumors (two-sided $\chi^{2}$ test $P=.015$ ) but not significantly more grade 3 tumors (two-sided $\chi^{2}$ test $P=.235$ ). DTH response was positive in $61.8 \%$ of patients treated 


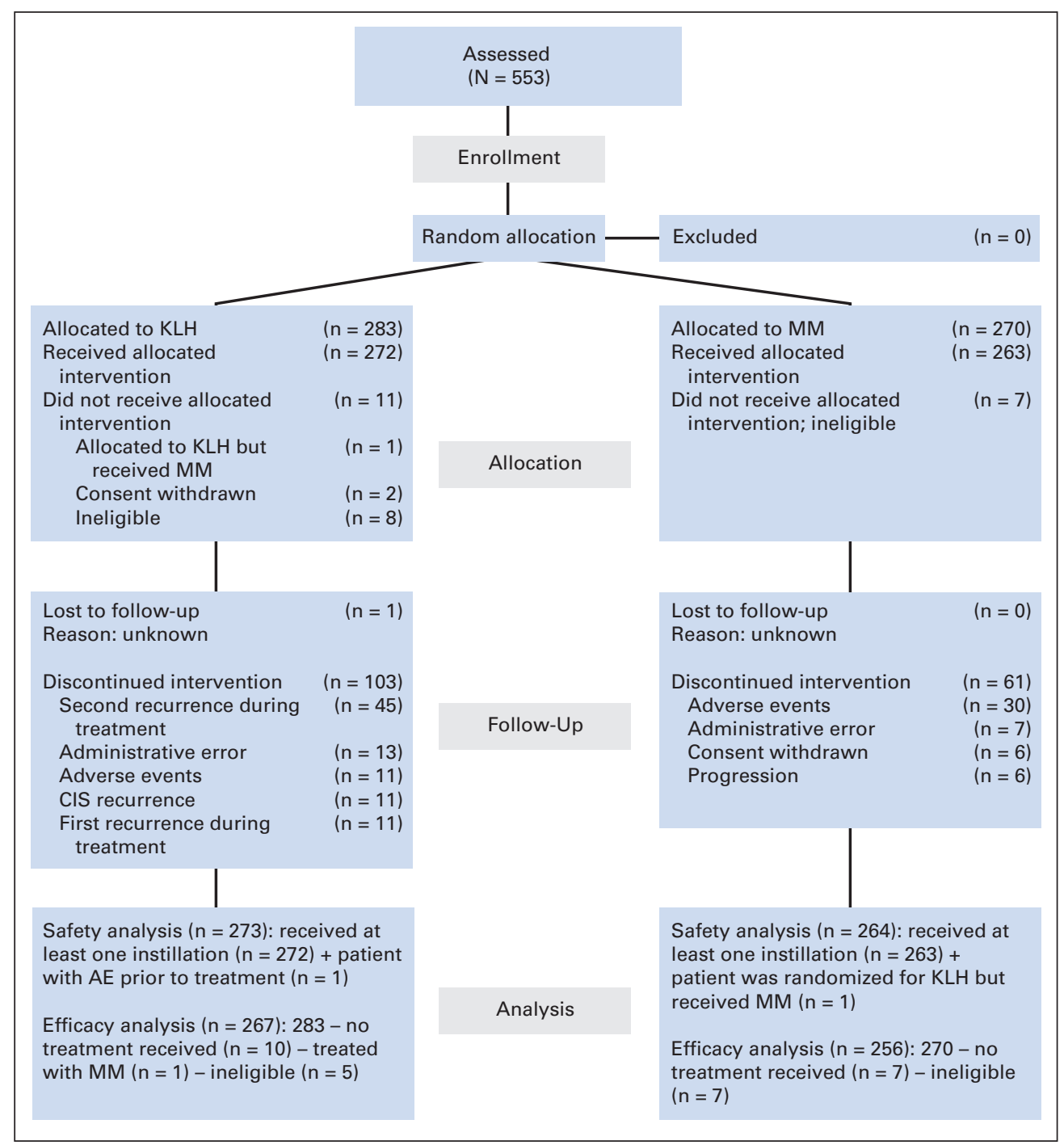

Fig 1. CONSORT diagram. $A E$, adverse event; CIS, carcinoma in situ; KLH, keyhole limpet hemocyanin; MM, mitomycin.

with KLH (165 of 267), negative in 97 patients (36.3\%), and unknown in five patients $(1.9 \%)$.

\section{Safety}

AEs were common ( $72 \%$ of all patients) but generally mild (56\%), with no differences between the KLH and MM arms (557 AEs $v 514$ AEs). No differences in frequency of AEs grouped in system classes were found except for two AE system classes. General disorders such as fever, flu-like symptoms, and fatigue occurred significantly more frequently in the KLH group (51 AEs $v 28$ AEs; two-sided $\chi^{2}$ test $P=.02)$. Allergic reactions and skin disorders such as eczema, rash, and itch occurred significantly more frequently in the MM group (53 AEs $v 9$ AEs; two-sided $\chi^{2}$ test $P<.001$ ). Significantly more mild AEs occurred in patients with a positive DTH response (two-sided $\chi^{2}$ test $P=.004$; Table 2).

\section{Efficacy}

In total, 163 patients (61\%) developed a recurrence after $\mathrm{KLH}$ instillations compared with 87 (34\%) after MM instillations (onesided Fisher's exact test $P<.001$ ). Median RFS in the KLH group was significantly shorter than that in the MM group (106 weeks [95\% CI, 86 to 126 weeks] $v 297$ weeks [ $95 \%$ CI, 202 to 392 weeks]; log-rank test $P<.001$; Fig 2). Some recurrences occurred outside the bladder: three recurrences in the MM group (once in the prostatic urethra; twice in the upper urinary tract) and four in the KLH group (all in the prostatic urethra).

Univariate Cox regression for RFS showed a crude HR of 2.26 for KLH (95\% CI, 1.74 to 2.94; $P<.001$; Table 3 ). Multivariate Cox regression showed an adjusted HR of 2.32 for KLH (95\% CI, 1.79 to $3.02 ; P<.001)$. Ratio of median RFS was 106 of 297 or $0.36(95 \% \mathrm{CI}$, 0.321 to 0.426$)$. Because 0.426 is far below the threshold of $0.75, \mathrm{KLH}$ is inferior to MM regarding RFS at a 0.001 level of significance.

Significantly more patients developed progression after MM $(\mathrm{n}=15)$ than after KLH $(\mathrm{n}=5$; one-sided Fisher's exact test; $P=$.015). Univariate analyses showed a possible relationship between treatment and progression chances in favor of KLH (log-rank test $P=.049$; univariate Cox regression HR, 0.38 [95\% CI, 0.14 to 1.04]; $P=.059$; Table 3 ). In the multivariate Cox regression analysis, this relationship was less obvious (adjusted HR, 0.43 [95\% CI, 0.15 to 1.17]; $P=.099$; Table 4). 


\begin{tabular}{|c|c|c|c|c|c|c|c|c|c|c|}
\hline \multirow[b]{2}{*}{ Characteristic } & \multicolumn{3}{|c|}{ KLH } & \multicolumn{3}{|c|}{ MM } & \multicolumn{3}{|c|}{ Total } & \multirow[b]{2}{*}{$P$} \\
\hline & No. & $\%$ & SD & No. & $\%$ & SD & No. & $\%$ & SD & \\
\hline No. of patients & 267 & & & 256 & & & 523 & & & \\
\hline Sex & & & & & & & & & & .910 \\
\hline Male & 218 & & & 210 & & & 428 & & & \\
\hline Female & 49 & & & 46 & & & 95 & & & \\
\hline Mean age, years & \multicolumn{2}{|c|}{66.9} & 10.3 & \multicolumn{2}{|c|}{67.7} & 9.3 & \multicolumn{2}{|c|}{67.3} & 9.8 & .428 \\
\hline History of CIS & 3 & & & 4 & & & 7 & & & .502 \\
\hline Tumor status & & & & & & & & & & .476 \\
\hline Primary & 144 & 54 & & 146 & 57 & & 290 & 55 & & \\
\hline Recurrent & 123 & 46 & & 110 & 43 & & 233 & 45 & & \\
\hline $\begin{array}{l}\text { Previous treatment in patients with recurrent tumors at } \\
\text { baseline }(n=233)\end{array}$ & & & & & & & & & & .086 \\
\hline Current single-post TURBT intravesical chemotherapy & 5 & 4 & & 2 & 2 & & 7 & 3 & & \\
\hline Previous single-post TURBT intravesical chemotherapy & 10 & 8 & & 3 & 3 & & 13 & 6 & & \\
\hline Local adjuvant intravesical therapy & 31 & 25 & & 27 & 25 & & 58 & 25 & & \\
\hline Other therapy & 3 & 2 & & 0 & 0 & & 3 & 1 & & \\
\hline No therapy other than TURBT & 74 & 60 & & 78 & 71 & & 152 & 65 & & \\
\hline Tumor stage & & & & & & & & & & .015 \\
\hline pTa & 216 & 81 & & 184 & 72 & & 400 & 76 & & \\
\hline pT1 & 51 & 19 & & 72 & 28 & & 123 & 24 & & \\
\hline Tumor grade & & & & & & & & & & .235 \\
\hline 1 & 88 & 33 & & 79 & 31 & & 167 & 32 & & \\
\hline 2 & 148 & 55 & & 134 & 52 & & 282 & 54 & & \\
\hline 3 & 31 & 12 & & 43 & 17 & & 74 & 14 & & \\
\hline No. of tumors & & & & & & & & & & .849 \\
\hline Single & 89 & 33 & & 83 & 32 & & 172 & 33 & & \\
\hline Multiple & 178 & 66 & & 172 & 67 & & 350 & 67 & & \\
\hline Unknown & 0 & & & 1 & 0 & & 1 & 0 & & \\
\hline Largest diameter, mm & 20 & & 29 & 19 & & 16 & 39 & & 24 & .496 \\
\hline
\end{tabular}

The mean number of recurrent tumors after KLH was 3.1 (standard deviation, 3.1) and 2.4 after MM (standard deviation, 2.2; onesided Mann-Whitney $U$ test $P=.154$ ). Recurrence rate was 2.32 after KLH and 1.05 after MM. Significantly more DTH-positive patients than DTH-negative patients developed a recurrence (two-sided $\chi^{2}$ test $P=.031$; Table 5), but RFS and number of recurrences were not significantly different (log-rank test $P=.163$ and two-sided $t$ test $P=.108$, respectively). Significantly more patients with a negative DTH response $(\mathrm{n}=4)$ than DTH-positive patients

\begin{tabular}{|c|c|c|c|c|c|c|c|}
\hline \multirow[b]{3}{*}{ Severity } & \multicolumn{6}{|c|}{ DTH Response } & \multirow[b]{3}{*}{ Total } \\
\hline & \multicolumn{2}{|c|}{ Positive } & \multicolumn{2}{|c|}{ Negative } & \multicolumn{2}{|c|}{ Unknown } & \\
\hline & No. & $\%$ & No. & $\%$ & No. & $\%$ & \\
\hline Mild & 207 & 64 & 111 & 50 & 1 & 12.5 & 319 \\
\hline Moderate & 96 & 30 & 89 & 39 & 6 & 75 & 189 \\
\hline Severe & 22 & 7 & 25 & 11 & 1 & 12.5 & 48 \\
\hline Unknown & 0 & 0 & 1 & 0 & 0 & 0 & 1 \\
\hline Total & 325 & & 224 & & 8 & & 557 \\
\hline \multicolumn{8}{|c|}{$\begin{array}{l}\text { NOTE. Patients with a positive delayed-type hypersensitivity (DTH) response } \\
\left.\text { had significantly more adverse events (two-sided } \chi^{2} \text { test } P=.004\right) \text {. }\end{array}$} \\
\hline
\end{tabular}

$(\mathrm{n}=1)$ showed progression (two-sided $\chi^{2}$ test $P=.044$ ), and time to progression tended to be shorter for DTH-negative patients (log-rank test $P=.051$ ).

For the subgroup analysis in patients with recurrent disease and a history of intravesical treatments, a total of 81 patients were identified (49 in the KLH group and 32 in the MM group). The number of patients with recurrences and time to recurrence was not statistically significantly different for the two treatment groups (one-sided Fisher's exact test $P=.194$ and $\log$-rank test $P=.558$, respectively).

\section{DISCUSSION}

In this prospective, randomized phase III trial, significantly more recurrences occurred after treatment with KLH compared with MM $(P<.001)$. Literature reporting on KLH and recurrence chances is diverse, and the number of patients is low. Jurincic et al ${ }^{10}$ treated 44 patients with either KLH (single IC preimmunization followed by an unknown number of monthly instillations with 10 mg KLH) or MM (unknown number of monthly instillations with $20 \mathrm{mg} \mathrm{MM}$ in $30 \mathrm{~mL}$ saline) and found significantly fewer recurrences after KLH treatment than after MM treatment $(P<.05)$. Kälble et $\mathrm{al}^{11}$ compared KLH (IC preimmunization followed by instillations [10 mg KLH in $20 \mathrm{~mL}$ saline] given once per week for 


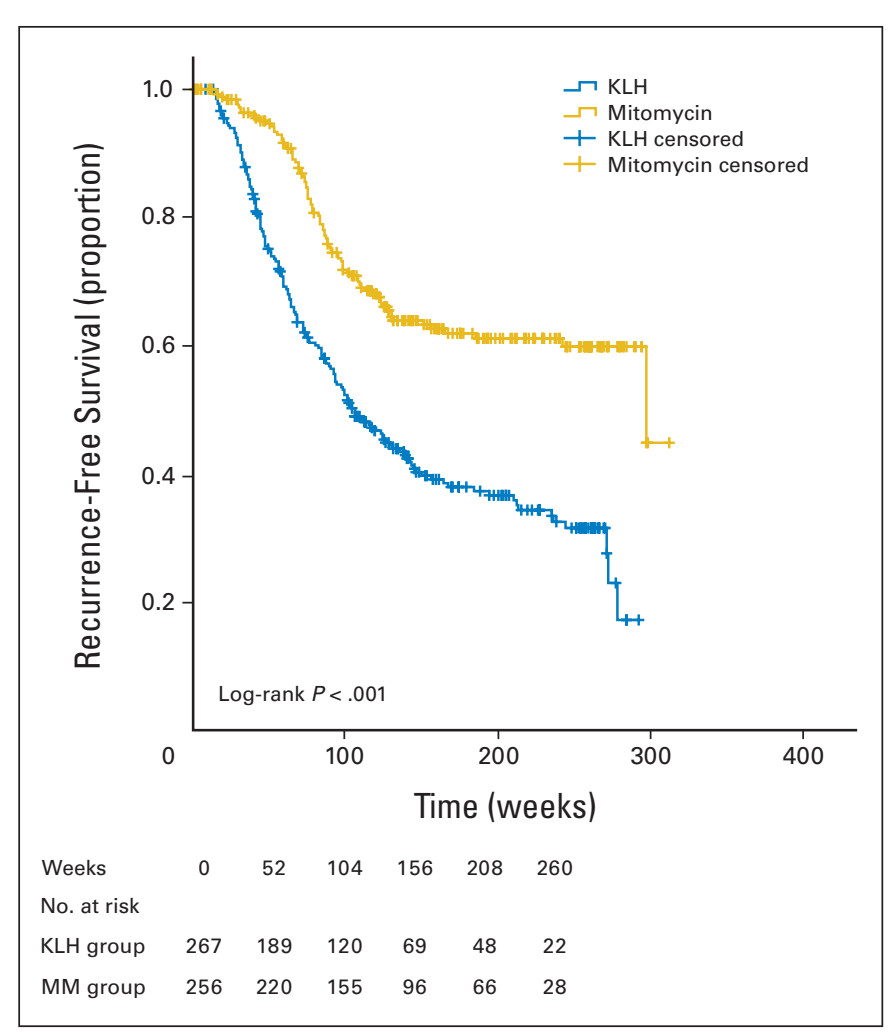

Fig 2. Kaplan-Meier curve for recurrence-free survival. KLH, keyhole limpet hemocyanin; MM, mitomycin.

6 weeks, once per month for the next 4 months, followed by two more instillations given at months 7 and 10) to BCG (instillations [120 mg BCG in $50 \mathrm{~mL}$ saline] given once per week for 6 weeks, once per month for the next 4 months, followed by two more instillations at months 7 and 10) in 38 patients and reported more recurrences after treatment with KLH (41.2\% v 14.3\% after BCG instillations). Jurincic-Winkler et $\mathrm{al}^{13}$ found that $11(52 \%)$ of 21 patients with CIS achieved a complete response after preimmunization and instillation with $20 \mathrm{mg}$ KLH in $20 \mathrm{mg}$ saline once per week for 2 weeks, of which five patients (24\%) remained CIS free during follow-up (mean follow-up, 31.7 months). The long-term outcome showed long-term tumor remission in only two pa- tients. ${ }^{14}$ Echarti et al $^{15}$ found a significantly longer RFS in 37 patients after KLH treatment compared with several other intravesical treatments $(P=.0012)$. However, treatment schedule and KLH dosage varied among these patients. Lamm et $\mathrm{al}^{6}$ treated 64 patients with CIS and/or residual tumor with KLH (IC preimmunization followed by instillations once per week for 6 weeks with $\mathrm{KLH}$ in increasing dosages: $0.4,2.0,10$, and $50 \mathrm{mg}$ ). Complete response was seen in 50\% of patients with CIS and in $20 \%$ of patients with residual tumor. Linn et $\mathrm{al}^{16}$ reviewed KLH efficacy and concluded that it appeared to be similar or better than intravesical chemotherapy. However, best dosage, treatment interval, and manner of application (intravesical, intralesional, or systemic) remains uncertain. In all, studies on KLH are small with a wide variety of inclusion criteria and treatment schedules and therefore diverse results.

In our study, progression was uncommon $(3.8 \% ; \mathrm{n}=20)$ since patients were observed until they developed a first recurrence, not until progression. Nevertheless, progression occurred significantly more often after MM treatment than after KLH treatment. Univariate Cox regression analysis for PFS showed no statistically significant difference between KLH and MM (HR, 0.38; 95\% CI, 0.14 to $1.04 ; P=.059)$. When correcting for confounding factors by including more pT1 tumors in the MM group, the statistical significance was even less obvious (adjusted HR, 0.43; 95\% CI, 0.15 to $1.17 ; P=.099)$. This suggests that the larger number of patients with pT1 tumors in the MM group might have introduced a bias. Because our results on progression are conflicting and paradoxical compared with the results of other studies, further research should focus on clarifying this.

There is little literature on progression after KLH. Jurincic et $\mathrm{al}^{10}$ found progression in three patients treated with MM versus in one patient treated with KLH. However, progression was defined as upgrading. No progression was found after KLH treatment in patients with CIS, but mean follow-up was only 23.5 months. ${ }^{11}$ Kälble et $\mathrm{al}^{11}$ found an increase in grade or stage in two patients (11.8\%) after KLH treatment versus no progression after BCG. As in other studies, ${ }^{6,10,11,13,15,16}$ we found generally mild AEs after KLH instillations.

In this study, the MM schedule was instillations once per week for 4 weeks and then once per month for 7 months, which was the schedule commonly in use when this trial was conducted. Currently,

\begin{tabular}{|c|c|c|c|c|c|c|}
\hline \multirow[b]{2}{*}{ Variable } & \multicolumn{3}{|c|}{ RFS } & \multicolumn{3}{|c|}{ PFS } \\
\hline & $\mathrm{HR}$ & $95 \% \mathrm{Cl}$ & $P$ & $\mathrm{HR}$ & $95 \% \mathrm{Cl}$ & $P$ \\
\hline Treatment (reference group, MM) & 2.26 & 1.74 to 2.94 & $<.001$ & 0.38 & 0.14 to 1.04 & .059 \\
\hline Sex (reference group, male) & 0.94 & 0.68 to 1.31 & .720 & 1.22 & 0.41 to 3.66 & .719 \\
\hline Prior recurrence rate (reference group, primary)* & 1.43 & 1.12 to 1.84 & .005 & 0.60 & 0.23 to 1.57 & .297 \\
\hline Tumor stage (reference group, Ta) & 0.99 & 0.73 to 1.35 & .967 & 3.85 & 1.59 to 9.33 & .003 \\
\hline Grade 2 tumor (reference group, grade 1) & 1.26 & 0.96 to 1.66 & .097 & 7.16 & 0.92 to 55.45 & .060 \\
\hline Grade 3 tumor (reference group, grade 1) & 0.88 & 0.57 to 1.36 & .562 & 19.89 & 2.49 to 159.03 & .005 \\
\hline Tumor size (reference group, $\leq 15 \mathrm{~mm}$ ) & 0.82 & 0.62 to 1.08 & .152 & 0.65 & 0.24 to 1.75 & .392 \\
\hline No. of tumors (reference group, single) $†$ & 1.94 & 1.45 to 2.58 & $<.001$ & 1.39 & 0.53 to 3.61 & .505 \\
\hline
\end{tabular}




\begin{tabular}{|c|c|c|c|c|c|c|}
\hline \multirow[b]{2}{*}{ Variable } & \multicolumn{3}{|c|}{ RFS } & \multicolumn{3}{|c|}{ PFS } \\
\hline & $H R$ & $95 \% \mathrm{Cl}$ & $P$ & $\mathrm{HR}$ & $95 \% \mathrm{Cl}$ & $P$ \\
\hline Treatment (reference group, MM & 2.32 & 1.79 to 3.02 & $<.001$ & 0.43 & 0.15 to 1.17 & .099 \\
\hline Prior recurrence rate (reference group, primary)* & 1.36 & 1.06 to 1.75 & .015 & N/A & & N/A \\
\hline Tumor stage (reference group, Ta) & N/A & & N/A & 2.13 & 0.82 to 5.56 & .121 \\
\hline Grade 2 tumor (reference group, grade 1) & N/A & & N/A & 5.66 & 0.71 to 44.90 & .101 \\
\hline Grade 3 tumor (reference group, grade 1) & N/A & & N/A & 11.76 & 1.34 to 103.53 & .026 \\
\hline No. of tumors (reference group, single)† & 1.88 & 1.41 to 2.51 & $<.001$ & $\mathrm{~N} / \mathrm{A}$ & & N/A \\
\hline
\end{tabular}

Abbreviations: HR, hazard ratio; MM, mitomycin; N/A, not applicable; PFS, progression-free survival; RFS, recurrence-free survival.

*Prior recurrence rate: primary $v$ recurrent.

†No. of tumors: single $v$ multiple tumors.

many different schedules are being used for MM, and guidelines do not recommend any particular schedule. ${ }^{5,17,18}$

Patients in this study had mainly intermediate or high risk of recurrences. The current guidelines advise BCG treatment for these patients, ${ }^{5,18}$ because randomized controlled trials and meta-analyses have shown BCG maintenance to be superior to MM in preventing recurrences. ${ }^{19,20}$ However, literature on superiority of BCG over MM for progression is less clear, as shown by Mälmstrom et al. ${ }^{20}$ This suggests that KLH is also inferior to BCG. Only Kälble et $\mathrm{al}^{11} \mathrm{com}$ pared BCG with KLH, and they indeed found that KLH is inferior to BCG.

Our results are contradictory on the need for preimmunization: a positive skin test appeared to be a prognostic factor for recurrences, but a negative DTH response was associated with progression. However, preimmunization was adequate in only $62 \%$ of the patients.

The exact mechanism behind KLH is not known. The most likely hypothesis is that KLH enhances the cellular immune system, ${ }^{21-25}$ which is the rationale behind preimmunization. Several studies reported on the need for preimmunization: Swerdlow et $\mathrm{al}^{26}$ found fewer tumors in mice preimmunized with KLH than in mice without preimmunization. However, the effect also depended on the intravesical KLH dose. Lamm et $\mathrm{al}^{27}$ found significantly reduced tumor incidence and tumor volume $(P<.001)$ and

\begin{tabular}{|c|c|c|c|c|c|c|c|}
\hline \multirow[b]{3}{*}{ Response } & \multicolumn{7}{|c|}{ DTH Response } \\
\hline & \multicolumn{2}{|c|}{ Positive } & \multicolumn{2}{|c|}{ Negative } & \multicolumn{2}{|c|}{ Unknown } & \multirow[b]{2}{*}{ Tota } \\
\hline & No. & $\%$ & No. & $\%$ & No. & $\%$ & \\
\hline \multicolumn{8}{|l|}{ Recurrence } \\
\hline Yes & 109 & 66 & 51 & 53 & 3 & 60 & 163 \\
\hline No & 56 & 34 & 46 & 47 & 2 & 40 & 104 \\
\hline Total & 165 & 100 & 97 & 100 & 5 & 100 & 267 \\
\hline \multicolumn{8}{|l|}{ Progression } \\
\hline Yes & 1 & 1 & 4 & 4 & 0 & 0 & 5 \\
\hline No & 164 & 99 & 93 & 96 & 5 & 100 & 262 \\
\hline Total & 165 & 100 & 97 & 100 & 5 & 100 & 267 \\
\hline
\end{tabular}

NOTE. A positive delayed-type hypersensitivity (DTH) is associated with a higher chance of recurrence (two-sided Pearson $\chi^{2}$ test $P=.031$ ). A negative DTH is associated with a higher chance of progression (two-sided Pearson $\chi^{2}$ test $P=.044$ ). significantly increased survival $(P=.001)$ of mice after immunization before tumor transplantation compared with immunization after tumor transplantation. Jurincic-Winkler et $\mathrm{al}^{25}$ found a relationship between positive skin test and fewer recurrences in only nine patients with primary NMIBC treated with intravesical KLH. As suggested by Oyelaran et al, ${ }^{28}$ differences in clinical responses with KLH may be due to differences in antibody profiles, which vary considerably from person to person. In all, the immunology behind KLH has not been clarified and needs further testing.

$\mathrm{KLH}$ is registered for prevention of bladder cancer after TURBT and after failure of established therapies. In a subgroup analysis with these patients $(\mathrm{n}=81)$, RFS was not significantly different between the KLH and MM arms. However, these results should be interpreted with care because subgroup analyses are prone to selection bias and, because of the small sample size, they are often underpowered. Therefore, no firm conclusions can be drawn from these subgroup analyses.

In daily urologic practice, NMIBC is sometimes coagulated instead of resected, but this means a lack of histologic confirmation. Therefore, additional analyses were done in which coagulated lesions, lack of biopsies, or lack of any resection were considered to be a protocol violation. Then, 210 patients developed a recurrence, with significantly more recurrences and significantly shorter RFS for KLH patients (both $P<.001$ ).

Sometimes in daily urologic practice, a recurrence is seen at cystoscopy but is not immediately resected. When considering the date at which the recurrence was first seen to be the date of recurrence, RFS was significantly shorter after KLH treatment than after MM treatment $(P<.001)$. When considering all lesions, whether pathologically confirmed, coagulated, or not resected at all, to be a recurrence, RFS was once more significantly shorter for patients treated with $\mathrm{KLH}(P<.001)$.

In conclusion, this prospective, randomized phase III trial shows that KLH had a safety profile different from that of MM. Patients with a positive skin test as a result of preimmunization with intracutaneous KLH had significantly more recurrences. KLH was inferior to MM in preventing NMIBC recurrences. KLH tended to prevent progression more effectively than MM, but when correcting for the higher number of patients with pT1 tumors, this could not be shown. More research is needed to clarify the immunologic effects of KLH and the effects of KLH on progression. 


\section{AUTHORS' DISCLOSURES OF POTENTIAL CONFLICTS} OF INTEREST

Although all authors completed the disclosure declaration, the following author(s) indicated a financial or other interest that is relevant to the subject matter under consideration in this article. Certain relationships marked with a " $U$ " are those for which no compensation was received; those relationships marked with a " $C$ " were compensated. For a detailed description of the disclosure categories, or for more information about ASCO's conflict of interest policy, please refer to the Author Disclosure Declaration and the Disclosures of Potential Conflicts of Interest section in Information for Contributors.

Employment or Leadership Position: None Consultant or Advisory Role: None Stock Ownership: None Honoraria: None Research
Funding: Wim P.J. Witjes, biosyn Arzneimittel GmbH Expert Testimony: None Other Remuneration: None

\section{AUTHOR CONTRIBUTIONS}

Conception and design: Wim P.J. Witjes, J. Alfred Witjes

Administrative support: Wim P.J. Witjes

Collection and assembly of data: Wim P.J. Witjes, Maria H.D.

Janzing-Pastors, Christien T.M. Caris

Data analysis and interpretation: Rianne J.M. Lammers, Wim P.J.

Witjes, Christien T.M. Caris, J. Alfred Witjes

Manuscript writing: All authors

Final approval of manuscript: All authors

\section{REFERENCES}

1. Jemal A, Bray F, Center MM, et al: Global cancer statistics. CA Cancer J Clin 61:69-90, 2011

2. Kirkali Z, Chan T, Manoharan M, et al: Bladder cancer: Epidemiology, staging and grading, and diagnosis. Urology 66:4-34, 2005

3. Sylvester RJ, van der Meijden AP, Oosterlinck $W$, et al: Predicting recurrence and progression in individual patients with stage Ta T1 bladder cancer using EORTC risk tables: A combined analysis of 2596 patients from seven EORTC trials. Eur Urol 49:466-475, 2006; discussion 475-477

4. Babjuk M, Oosterlinck W, Sylvester R, et al: EAU guidelines on non-muscle-invasive urothelial carcinoma of the bladder. Eur Urol 54:303-314, 2008

5. Hall MC, Chang SS, Dalbagni G, et al: Guideline for the management of nonmuscle invasive bladder cancer (stages Ta, T1, and Tis): 2007 update. J Urol 178:2314-2330, 2007

6. Lamm DL, Dehaven JI, Riggs DR: Keyhole limpet hemocyanin immunotherapy of bladder cancer: Laboratory and clinical studies. Eur Urol 37:4144, 2000

7. Shelley MD, Court JB, Kynaston $H$, et al: Intravesical bacillus Calmette-Guerin versus mitomycin C for Ta and T1 bladder cancer. Cochrane Database Syst Rev 3:CD003231, 2003

8. Harris JR, Markl J: Keyhole limpet hemocyanin (KLH): A biomedical review. Micron 30:597-623, 1999

9. Olsson CA, Chute R, Rao CN: Immunologic reduction of bladder cancer recurrence rate. J Urol 111:173-176, 1974

10. Jurincic $C D$, Engelmann U, Gasch J, et al: Immunotherapy in bladder cancer with keyholelimpet hemocyanin: A randomized study. J Urol 139:723-726, 1988

11. Kälble $T$, Möhring $K$, Ikinger $U$, et al: Intravesikal Rezidivprophylaxe beim oberflächlichen Harnblasenkarzinom mit BCG und KLH. [Intravesical prevention of recurrence of superficial urinary bladder cancer with BCG and KLH. A prospective randomized study] [In German]. Urologe A 30:118-121, 1991

12. Flamm J, Bucher A, Höltl W, et al: Recurrent superficial transitional cell carcinoma of the bladder: Adjuvant topical chemotherapy versus immunotherapy-A prospective randomized trial. J Urol 144:260-263, 1990

13. Jurincic-Winkler $C$, Metz KA, Beuth J, et al: Effect of keyhole limpet hemocyanin (KLH) and bacillus Calmette-Guérin (BCG) instillation on carcinoma in situ of the urinary bladder. Anticancer Res 15:2771-2776, 1995

14. Jurincic-Winkler $C D$, Metz KA, Beuth J, et al: Keyhole limpet hemocyanin for carcinoma in situ of the bladder: A long-term follow-up study. Eur Urol 37:45-49, 2000

15. Echarti C, Jurincic-Winkler CD, Klippel KF: Efficacy of prophylactic Immucothel in patients pretreated with conventional drugs to prevent recurrence of superficial bladder carcinoma. Eur Urol 37:50-53, 2000

16. Linn JF, Black P, Derksen K, et al: Keyhole limpet haemocyanin in experimental bladder cancer: Literature review and own results. Eur Urol 37:3440, 2000

17. Sylvester RJ: Intravesical chemotherapy in non-muscle-invasive bladder cancer: What schedule and duration of treatment? Eur Urol 52:951-953, 2007; discussion 953-954

18. Babjuk M, Oosterlinck W, Sylvester R, et al: EAU Guidelines on non-muscle-invasive urothelial carcinoma of the bladder, the 2011 Update. Eur Urol 59:997-1008, 2011

19. Böhle A, Jocham D, Bock PR: Intravesical bacillus Calmette-Guerin versus mitomycin $C$ for superficial bladder cancer: A formal meta-analysis of comparative studies on recurrence and toxicity. J Urol 169:90-95, 2003

20. Malmström PU, Sylvester RJ, Crawford DE, et al: An individual patient data meta-analysis of the long-term outcome of randomised studies comparing intravesical mitomycin $\mathrm{C}$ versus bacillus Calmette-Guérin for nonmuscle-invasive bladder cancer. Eur Urol 56:247-256, 2009

21. Moltedo $B$, Faunes $F$, Haussmann $D$, et al: Immunotherapeutic effect of Concholepas hemocyanin in the murine bladder cancer model: Evidence for conserved antitumor properties among hemocyanins. J Urol 176:2690-2695, 2006

22. Moltó LM, Carballido J, Jurincic $C$, et al: Keyhole limpet hemocyanine can enhance the natural killer activity of patients with transitional cell carcinoma of the bladder. Eur Urol 19:74-78, 1991

23. Jurincic-Winkler $C$, Metz KA, Beuth J, et al: Immunohistological findings in patients with superficial bladder carcinoma after intravesical instillation of keyhole limpet haemocyanin. Br J Urol 76:702707, 1995

24. Jurincic-Winkler CD, Gallati $H$, Alvarez-Mon $M$, et al: Urinary interleukin-1 alpha levels are increased by intravesical instillation with keyhole limpet hemocyanin in patients with superficial transitional cell carcinoma of the bladder. Eur Urol 28:334-339, 1995

25. Jurincic-Winkler $C D$, von der Kammer $H$, Beuth J, et al: Antibody response to keyhole limpet hemocyanin (KLH) treatment in patients with superficial bladder carcinoma. Anticancer Res 16:21052110, 1996

26. Swerdlow RD, Ratliff TL, La Regina $M$, et al: Immunotherapy with keyhole limpet hemocyanin: Efficacy and safety in the MB-49 intravesical murine bladder tumor model. J Urol 151:1718-1722, 1994

27. Lamm DL, DeHaven JI, Riggs DR, et al: Immunotherapy of murine bladder cancer with keyhole limpet hemocyanin (KLH). J Urol 149:648-652, 1993

28. Oyelaran O, Gildersleeve JC: Evaluation of human antibody responses to keyhole limpet hemocyanin on a carbohydrate microarray. Proteomics Clin Appl 4:285-294, 2010 